\title{
THE LOCAL HURWITZ CONSTANT AND DIOPHANTINE APPROXIMATION ON HECKE GROUPS
}

\section{J. LEHNER}

Abstract. Define the Hecke group by

$$
G_{q}=\left\langle\left(\begin{array}{cc}
1 & \lambda_{q} \\
0 & 1
\end{array}\right),\left(\begin{array}{cc}
0 & -1 \\
1 & 0
\end{array}\right)\right\rangle
$$

$\lambda_{q}=2 \cos \pi / q, q=3,4, \ldots$ We call $G_{q}(\infty)$ the $G_{q}$-rationals, and $\mathbb{R}-$ $G_{q}(\infty)$ the $G_{q}$-irrationals. The problem we treat here is the approximation of $G_{q}$-irrationals by $G_{q}$-rationals. Let $M(\alpha)$ be the upper bound of numbers $c$ for which $|\alpha-k / m|<1 / \mathrm{cm}^{2}$ for all $G_{q}$-irrationals and infinitely many $k / m \in G_{q}(\infty)$. Set $h_{q}^{\prime}=\inf _{\alpha} M(\alpha)$. We call $h_{q}^{\prime}$ the Hurwitz constant for $G_{q}$. It is known that $h_{q}^{\prime}=2, q$ even; $h_{q}^{\prime}=2\left(1+\left(1-\lambda_{q} / 2\right)^{2}\right)^{1 / 2}, q$ odd. In this paper we prove this result by using $\lambda_{q}$-continued fractions, as developed previously by $\mathrm{D}$. Rosen. Write

$$
\alpha-\frac{P_{n-1}}{Q_{n-1}}=\frac{(-1)^{n-1} \varepsilon_{1} \varepsilon_{2} \cdots \varepsilon_{n}}{m_{n-1}(\alpha) Q_{n-1}^{2}},
$$

where $\varepsilon_{i}= \pm 1$ and $P_{i} / Q_{i}$ are the convergents of the $\lambda_{q}$-continued fraction for $\alpha$. Then $M(\alpha)=\varlimsup_{n} m_{n}(\alpha)$. We call $m_{n}(\alpha)$ the local Hurwitz constant. In the final section we prove some results on the local Hurwitz constant. For example (Theorem 4), it is shown that if $q$ is odd and $\varepsilon_{n+1}=\varepsilon_{n+2}=+1$, then $m_{i} \geq\left(\lambda_{q}^{2}+4\right)^{1 / 2}>h_{q}^{\prime}$ for at least one of $i=n-1, n, n+1$.

\section{INTRODUCTION}

Let the Hecke group

$$
G_{q}=\left\langle\left(\begin{array}{cc}
1 & \lambda_{q} \\
0 & 1
\end{array}\right),\left(\begin{array}{cc}
0 & -1 \\
1 & 0
\end{array}\right)\right\rangle, \quad \lambda_{q}=2 \cos \frac{\pi}{q}, q \geq 3,
$$

act on the upper half-plane $\operatorname{Im} z>0$ by Möbius transformations $z \rightarrow$ $(k z+l) /(m z+n),\left(\begin{array}{cc}k & l \\ m & n\end{array}\right) \in G_{q} . G_{q}$ is a horocyclic group with cusp set $G_{q}(\infty)$, which are called $G_{q}$-rationals. The points of $\mathbb{R}-G_{q}(\infty)$ are the $G_{q^{-}}$ irrationals. In [4] we considered the problem of approximating a $G_{q}$-irrational by $G_{q}$-rationals.

When $q=3, G_{q}$ becomes the classical modular group $\operatorname{PSL}(2, \mathbb{Z})$ and we are considering classical Diophantine approximation of rationals by irrationals.

Received August 31, 1988; revised August 21, 1989.

1980 Mathematics Subject Classification (1985 Revision). Primary 11F03. 
A. Hurwitz showed that when $\alpha$ is irrational, there exist infinitely many reduced fractions $k / m$ for which

$$
\left|\alpha-\frac{k}{m}\right|<\frac{1}{\sqrt{5} m^{2}},
$$

where $\sqrt{5}$ is the best constant possible. From now on we consider only $q \geq 4$.

Let $\alpha$ be $G_{q}$-irrational and suppose

$$
\left|\alpha-\frac{k}{m}\right|<\frac{1}{c m^{2}}, \quad \frac{k}{m} \in G_{q}(\infty), m>0 .
$$

We denote by $M(\alpha)$ the upper bound of numbers $c$ for which (1.1) holds for infinitely many $\mathrm{k} / \mathrm{m}$ and put

$$
h_{q}^{\prime}=\inf _{\alpha} M(\alpha), \quad \alpha G_{q} \text {-irrational. }
$$

We call $h_{q}^{\prime}$ the Hurwitz constant for $G_{q}$. In [4] we proved that $h_{q}^{\prime}=2$ when $q$ is even and gave bounds for $h_{q}^{\prime}$ when $q$ is odd. In [3] A. Haas and C. Series found the exact value of $h_{q}^{\prime}$. So we now know that $h_{q}^{\prime}=h_{q}$, where $h_{q}$ is defined by

$$
h_{q}=\left\{\begin{array}{l}
2, \quad q \text { even }, \geq 4, \\
2\left(1+\left(1-\lambda_{q} / 2\right)^{2}\right)^{1 / 2}, \quad q \text { odd. }
\end{array}\right.
$$

(Note that the notation of [3] differs from ours-their $h_{q}$ is the reciprocal of ours-and the methods of the two papers are quite different.)

From now on we write $G$ for $G_{q}$, and $\lambda$ for $\lambda_{q}$. In [4] we made use of a type of continued fraction expansion of the limit set of $G_{q}$, i.e., of $\mathbb{R}$, developed by D. Rosen [5]. (This limit set was also studied by Thea Pignataro in her Princeton thesis (1984, unpublished).) This expansion is called a (reduced) $\lambda$-fraction and represents every real number $\alpha$ uniquely:

$$
\alpha \equiv \alpha_{0}=r_{0} \lambda+\frac{\varepsilon_{1}}{r_{1} \lambda+\cdots}=\left[r_{0} \lambda, \frac{\varepsilon_{1}}{r_{1} \lambda}, \ldots\right] .
$$

Here $\varepsilon_{i}= \pm 1, r_{0}=r_{0}\left(\alpha_{0}\right)$ is an integer, $r_{i}=r_{i}\left(\alpha_{0}\right), i \geq 1$, are positive integers, and certain conditions are placed on the $\varepsilon_{i}$ and $r_{i}$. The above expansion, referred to as $\lambda \mathrm{CF} \alpha_{0}$, is finite if and only if $\alpha_{0}$ is $G$-rational. Denote the convergents of (1.4) by

$$
\frac{P_{n}}{Q_{n}}=\left[r_{0} \lambda, \ldots, \frac{\varepsilon_{n}}{r_{n} \lambda}\right], \quad Q_{0}=1 .
$$

Our general plan of attack follows Hurwitz and was described in [4] at the beginning of $\S 3$. Hurwitz first shows that if (1.1) is satisfied by any rational number $P / Q$ in lowest terms, then $P / Q$ must be a convergent in the expansion of $\alpha$ as a regular continued fraction. The problem is thus reduced to studying the approximation of $\alpha$ by its convergents.

Here we follow a similar plan. By a preliminary theorem [4, Theorem 3] the approximation of a $G$-irrational $\alpha_{0}$ by $G$-rationals was reduced to the 
approximation of $\alpha_{0}$ by the convergents $P_{n} / Q_{n}$ of $\lambda \mathrm{CF} \alpha_{0}$. Thus the inequality (1.1) was replaced by an inequality derived from

$$
\alpha_{0}-\frac{P_{n-1}}{Q_{n-1}}=\frac{(-1)^{n-1} \varepsilon_{1} \varepsilon_{2} \cdots \varepsilon_{n}}{m_{n-1} Q_{n-1}^{2}}, \quad m_{n-1}=m_{n-1}\left(\alpha_{0}\right),
$$

and the object of study was $m_{n-1}\left(\alpha_{0}\right)$. Clearly,

$$
M\left(\alpha_{0}\right)=\varlimsup_{n \rightarrow \infty} m_{n-1}\left(\alpha_{0}\right), \quad h_{q}=\inf _{\alpha_{0}} M\left(\alpha_{0}\right) .
$$

We call $m_{n}\left(\alpha_{0}\right)$ a local Hurwitz constant.

Two $\lambda$ CF $\alpha$ and $\beta$ are said to be equivalent, and we write $\alpha \sim \beta$, if their expansions agree from a certain point on. It is easy to check that $\alpha \sim \beta$ if and only if $\alpha= \pm V \beta$ for a $V \in G$. It is clear that

$$
\alpha \sim \beta \Rightarrow M(\alpha)=M(\beta) .
$$

The object of the present paper is to provide inequalities for the local Hurwitz constants. First, however, we shall prove that the Hurwitz constant $h_{q}^{\prime}$ has the value $h_{q}$ in (1.3), using the method of $\lambda$-fractions. The result follows from

Theorem 1. Let $\alpha_{0}$ be a G-irrational given by (1.4). When $q$ is odd,

$$
M\left(\alpha_{0}\right) \geq 2\left(1+(1-\lambda / 2)^{2}\right)^{1 / 2},
$$

with equality if and only if

$$
\alpha_{0} \sim 1-\lambda / 2+\left(1+(1-\lambda / 2)^{2}\right)^{1 / 2} .
$$

When $q$ is even, $M\left(\alpha_{0}\right) \geq 2$, with equality if and only if $\alpha_{0} \sim 1$.

Of course, knowledge of the value of $h_{q}, q$ odd, given in [3], was of the greatest value in constructing the proof.

The local Hurwitz constants are also discussed. Let $m_{n-1} \equiv m_{n-1}(\alpha)$ be defined by (1.6).

Theorem 2. If $\varepsilon_{n+1}=1$, then $m_{n-1}>2, m_{n}<2$, or $m_{n-1}<2, m_{n}>2$.

Theorem 3. Let $q$ be odd. If $r_{n} \geq 2$ and $\varepsilon_{n-1}=1$, then $m_{n-1} \geq h_{q}$.

Theorem 4. Let $q$ be odd. If $\varepsilon_{n+1}=\varepsilon_{n+2}=1$, then $m_{i} \geq\left(\lambda^{2}+4\right)^{1 / 2}>h_{q}$ for at least one of $i=n-1, n, n+1$.

\section{Definitions AND basic Lemmas}

In this section we gather together definitions and theorems needed in the sequel; most of these can be found in [5 and 4]. Let $q \geq 4$. With the notations of (1.4), (1.5) we have

$$
\begin{aligned}
P_{n} & =r_{n} \lambda P_{n-1}+\varepsilon_{n} P_{n-2}, \quad n \geq 1, \\
Q_{n} & =r_{n} \lambda Q_{n-1}+\varepsilon_{n} Q_{n-2}, \quad
\end{aligned}
$$


where

$$
\begin{gathered}
P_{-1}=1, \quad P_{0}=r_{0} \lambda, \quad Q_{-1}=0, \quad Q_{0}=1, \\
P_{n} Q_{n-1}-P_{n-1} Q_{n}=(-1)^{n-1} \varepsilon_{1} \varepsilon_{2} \cdots \varepsilon_{n}, \quad n \geq 1, \\
\alpha-\frac{P_{n-1}}{Q_{n-1}}=(-1)^{n-1} \frac{\varepsilon_{1} \cdots \varepsilon_{n}}{m_{n-1}(\alpha) Q_{n-1}^{2}} .
\end{gathered}
$$

Here,

$$
\begin{gathered}
m_{n-1}(\alpha) \equiv m_{n-1}=\alpha_{n}+\varepsilon_{n} / \alpha_{n-1}^{\prime}, \quad n \geq 3, \\
\alpha_{n}=\left[r_{n} \lambda, \frac{\varepsilon_{n+1}}{r_{n+1} \lambda}, \ldots\right], \quad n \geq 0 ; \\
\alpha_{n-1}^{\prime}=\left[r_{n-1} \lambda, \frac{\varepsilon_{n-1}}{r_{n-2} \lambda}, \ldots, \frac{\varepsilon_{2}}{r_{1} \lambda}\right] .
\end{gathered}
$$

As we shall see later, $Q_{n} \geq 1$ and $m_{n-1}(\alpha)>0$. Note that $P_{n} / Q_{n}$ is a strictly decreasing sequence when all $\varepsilon_{i}=-1$. The periodic $\lambda \mathrm{CF}$ of period $p$,

$$
\alpha=\left[r_{0} \lambda, \frac{\varepsilon_{1}}{r_{1} \lambda}, \ldots, \frac{\varepsilon_{p-1}}{r_{p-1} \lambda}, \frac{\varepsilon_{p}}{r_{0} \lambda}, \frac{\varepsilon_{1}}{r_{1} \lambda}, \ldots\right]
$$

can be written as

$$
\alpha=\left[r_{0} \lambda, \overline{\frac{\varepsilon_{1}}{r_{1} \lambda}, \ldots, \frac{\varepsilon_{p-1}}{r_{p-1} \lambda}, \frac{\varepsilon_{p}}{r_{0} \lambda}}\right] \text {, }
$$

or as

$$
\alpha=\left[r_{0} \lambda, \frac{\varepsilon_{1}}{r_{1} \lambda}, \ldots, \frac{\varepsilon_{p-1}}{r_{p-1} \lambda}, \frac{\varepsilon_{p}}{\alpha}\right] .
$$

The following lemma is slightly more general than [5, p. 556].

Lemma 1. Let

$$
\alpha_{n \nu}=\left[b_{n}, \varepsilon_{n+1} / b_{n+1}, \ldots, \varepsilon_{\nu} / b_{\nu}\right]
$$

and

$$
\alpha_{n \nu}^{\prime}=\left[b_{n}^{\prime},-1 / b_{n+1}^{\prime}, \ldots,-1 / b_{\nu}^{\prime}\right]
$$

have $b_{\mu}, b_{\mu}^{\prime}>0,0 \leq n \leq \mu<\nu$. If $b_{\mu} \geq b_{\mu}^{\prime}$, then $\alpha_{n \nu} \geq \alpha_{n \nu}^{\prime}$, and $\alpha_{n \nu}>\alpha_{n \nu}^{\prime}$ if some $b_{\mu}>b_{\mu}^{\prime}$. If

$$
\alpha_{n}=\left[b_{n}, \varepsilon_{n+1} / b_{n+1}, \ldots\right]
$$

and

$$
\alpha_{n}^{\prime}=\left[b_{n}^{\prime},-1 / b_{n+1}^{\prime}, \ldots\right]
$$

are convergent fractions, and $b_{\mu} \geq b_{\mu}^{\prime}, \mu \geq n$, then $\alpha_{n} \geq \alpha_{n}^{\prime}$.

For $q$ odd, write $q=2 l-1, l \geq 3$; for $q$ even, write $q=2 l, l \geq 2$. Let

$$
s=[(q-3) / 2]=l-2, \quad l \geq 2 \text {. }
$$


The notation $(-1 / r \lambda)^{n}$ means a block of $n$ consecutive terms $-1 / r \lambda$. We shall frequently need the $\lambda \mathrm{CF}$

$$
B(n)=\left[\lambda,(-1 / \lambda)^{n-1}\right], \quad n \geq 2, \quad B(1)=\lambda,
$$

with $n$ partial quotients. Thus $[5$, p. 556],

$$
B(n+1)=\lambda-1 / B(n), \quad 1 \leq n \leq s+1,
$$

$B(n)$ is strictly decreasing,

$B(s)=1 /(\lambda-1), \quad B(s+1)=1, \quad q$ odd,

$$
B(s)=\lambda /\left(\lambda^{2}-2\right), \quad B(s+1)=2 / \lambda, \quad q \text { even. }
$$

Also let

$$
C(n)=\left[2 \lambda,(-1 / 2 \lambda)^{n-1}\right], \quad n \geq 2, \quad C(1)=2 \lambda .
$$

Then

$$
C(n+1)=2 \lambda-1 / C(n), \quad n \geq 1,
$$

$$
C(n) \text { is strictly decreasing, }
$$

$$
\lim _{n \rightarrow \infty} C(n)=\lambda+\left(\lambda^{2}-1\right)^{1 / 2} \text {. }
$$

We have

(2.8) $\left[C(n+1),-\frac{1}{T}\right]>\left[C(n),-\frac{1}{T}\right], \quad n \geq 1,0<T<\lambda+\left(\lambda^{2}-1\right)^{1 / 2}$.

Indeed, by Lemma 1 ,

$$
\left[C(n+1),-\frac{1}{T}\right]=\left[C(n),-\frac{1}{2 \lambda-1 / T}\right]>\left[C(n),-\frac{1}{T}\right],
$$

since $T+1 / T<\lambda+\left(\lambda^{2}-1\right)^{1 / 2}+\lambda-\left(\lambda^{2}-1\right)^{1 / 2}=2 \lambda$. Similarly,

$$
[B(k),-1 / T]>[B(k+1),-1 / T], \quad k \leq s, T>0 .
$$

In fact,

$$
\begin{aligned}
{\left[B(k),-\frac{1}{T}\right] } & >\left[B(k),-\frac{1}{\lambda-1 / T}\right] \\
& =\left[B(k)-\frac{1}{\lambda},-\frac{1}{T}\right]=\left[B(k+1),-\frac{1}{T}\right],
\end{aligned}
$$

since $T+1 / T \geq 2>\lambda$.

When $\lambda$ CF $\alpha$ is reduced (see $\S \S 3$ and 5 for the definition), we have

$$
\alpha_{n \nu} \geq 2 / \lambda, \quad \nu \geq n ; \quad \alpha_{n} \geq 2 / \lambda \quad \text { if } r_{0} \geq 1 \text { [5, Lemma 2], }
$$

where

$$
\begin{aligned}
& \alpha_{n \nu}=\left[r_{n} \lambda, \frac{\varepsilon_{n+1}}{r_{n+1} \lambda}, \ldots, \frac{\varepsilon_{\nu}}{r_{\nu} \lambda}\right], \quad \nu>n ; \quad \alpha_{n n}=r_{n} \lambda, \\
& \alpha_{n}=\left[r_{n} \lambda, \frac{\varepsilon_{n+1}}{r_{n+1} \lambda}, \ldots\right], \\
& Q_{n} \geq Q_{n-1}, \quad n \geq 1[5, \text { Theorem 3]. }
\end{aligned}
$$


Using these inequalities in (2.4) and (2.1), we get

$$
m_{n-1}(\alpha) \geq \frac{2}{\lambda}-1>0, \quad n \geq 3 ; \quad Q_{n} \geq 1, \quad n \geq 0,
$$

as stated earlier.

\section{Evaluation of the Hurwitz constant}

In this section our object is to prove Theorem 1. The result for even $q$ having been established in [4, Theorem 1], we now assume $q$ odd.

A $\lambda \mathrm{CF} \alpha_{0}=\left[r_{0} \lambda, \varepsilon_{1} / r_{1} \lambda, \ldots\right]$ is said to be reduced [5, p. 555] if

The inequality $r_{i} \lambda+\varepsilon_{i+1}<1$ (i.e., $r_{i}=1, \varepsilon_{i+1}=-1$ ) is satisfied for no more than $s$ consecutive values $i=j, j+1, \ldots, j+$ $s-1, j \geq 1$. Here $s$ is defined in (2.5a).

If $r_{i} \lambda+\varepsilon_{i+1}<1$ is satisfied for $s$ consecutive values $i=$ $j, \ldots, j+s-1$, then $r_{j+s} \geq 2$.

If $[B(s),-1 / 2 \lambda,-1 / B(s)]$ occurs, the succeeding $\varepsilon$ is +1 .

A reduced $\lambda \mathrm{CF}$ has the following properties, in addition to (2.9) and (2.10):

An infinite reduced $\lambda \mathrm{CF}$ converges.

Every real number $\alpha$ can be expanded uniquely by the "nearest integer algorithm" in a reduced $\lambda C F$. If the fraction is infinite, it converges to $\alpha$.

From now on, $\lambda \mathrm{CF}$ shall mean reduced $\lambda \mathrm{CF}$. Bear in mind that at this point we are interested in $\varlimsup \lim m_{n-1}\left(\alpha_{0}\right)$ rather than $m_{n-1}\left(\alpha_{0}\right)$ itself, because of (1.7).

We first consider the $\lambda \mathrm{CF} \alpha_{0}$ with all $\varepsilon_{\nu}=-1$. In $\alpha_{0}$, some terms $-1 / r \lambda$, $r \geq 2$, must occur by $(3,1)$; in fact, there is at least one such term in every block of length $s+1$. We shall make a series of transformations in $\lambda \mathrm{CF} \alpha_{0}$, each having the effect of decreasing $\alpha_{0}$ while leaving it reduced. The first transformation is to replace each $r_{\nu}>2$ by $r_{\nu}=2$, which by Lemma 1 decreases $\alpha_{0}$. For convenience let $r_{0}=2$, so that now

$$
\alpha_{0}=\left[C\left(t_{1}\right),-1 / B\left(u_{1}\right),-1 / C\left(t_{2}\right), \ldots\right], \quad t_{i} \geq 1,1 \leq u_{i} \leq s,
$$

by (3.1). By (2.8) we can assume further that $t=1$ or 2 .

The case $q=5$ is simpler to treat than the higher values of $q$. Let $\lambda=\lambda_{5}$; then $s=1$, so $u_{i}=1$. Moreover, $t_{i} \geq 2$ for all $i \geq 2$, otherwise (3.3) is 
violated. Thus, we decrease $\alpha_{0}$ by assuming $t_{i}=2$, and we shall temporarily assume $t_{1}=1$. Hence,

$$
\alpha_{0} \geq\left[2 \lambda, \overline{-\frac{1}{\lambda},-\frac{1}{2 \lambda},-\frac{1}{2 \lambda}} ;-\frac{1}{\lambda}\right]=: \tau_{0}=\tau_{3 n},
$$

a periodic $\lambda \mathrm{CF}$ of period 3 . If any $t_{j}$ is greater than 2 , we have strict inequality.

The reverse $\alpha_{3 n-1}^{\prime}$ can be extended to a periodic fraction with a decrease in value. This fraction, still denoted by $\alpha_{3 n-1}^{\prime}$, obviously satisfies

$$
\alpha_{3 n-1}^{\prime} \geq\left[2 \lambda,-\frac{1}{\lambda},\left(-\frac{1}{2 \lambda}\right)^{2}, \ldots\right]=\tau_{0} .
$$

Therefore,

$$
m_{3 n-1} \geq \tau_{0}-1 / \tau_{0} .
$$

By similar calculations we can show that

$$
m_{3 n-2} \geq \tau_{2}-\frac{1}{\tau_{3 n+1}^{\prime}}=2 \lambda-\frac{1}{\tau_{0}}+\tau_{0}-2 \lambda=\tau_{0}-\frac{1}{\tau_{0}},
$$

where we used $\tau_{3 n+1}^{\prime}=\tau_{3 n+1}=\tau_{1},-1 / \tau_{1}=\tau_{0}-2 \lambda$. Thus, $m_{3 n-1}$ and $m_{3 n-2}$ are both bounded below by $\tau_{0}-1 / \tau_{0}$. On the other hand,

$$
m_{3 n}=\tau_{3 n+1}-1 / \tau_{3 n}^{\prime}<\tau_{3 n+1}-\tau_{1}=\lambda-\cdots<\lambda .
$$

It remains to evaluate $\tau_{0}-1 / \tau_{0}$. Now, $\tau_{0}=2 \lambda-1 / \tau_{1}$, and it was shown in $\left[4\right.$, p. 126] that $\tau_{1}$ satisfies

$$
\tau_{1}^{2}-\lambda \tau_{1}+\frac{2 \lambda-1}{5}=0,
$$

where we used $\lambda^{2}-\lambda-1=0$. From this we calculate that

$$
\tau_{0}^{2}+(2-3 \lambda) \tau_{0}+1=0,
$$

or

$$
\tau_{0} \sim 1-\lambda / 2+\left(1+(1-\lambda / 2)^{2}\right)^{1 / 2} .
$$

Let $\tau_{0}^{*}$ be the other root, $\tau_{0} \tau_{0}^{*}=1$. Then,

$$
\begin{aligned}
m_{3 n-1}, m_{3 n-2} & \geq \tau_{0}-\frac{1}{\tau_{0}}=\tau_{0}-\tau_{0}^{*}=\left(9 \lambda^{2}-12 \lambda\right)^{1 / 2} \\
& =(9-3 \lambda)^{1 / 2}=2\left(1+\left(1-\frac{\lambda}{2}\right)^{2}\right)^{1 / 2}=h_{5} .
\end{aligned}
$$

From (3.10), (3.12), and (3.13) it follows that $M\left(\tau_{0}\right)=h_{5}$ when $\tau_{0}$ satisfies (3.12), and this is the only case of equality. Theorem 1 is now proved for $q=5$. 
We next assume $q \geq 7$. The case $t_{i}=2$ for some $i$ in (3.8) is not difficult. Suppose $B\left(u_{1}\right),-1 / 2 \lambda,-1 / 2 \lambda,-1 / B\left(u_{2}\right)$ occurs. Setting $\left[2 \lambda,-1 / B\left(u_{2}\right), \ldots\right]$ $=\left[r_{n} \lambda, \ldots\right]$, we have $\alpha_{n} \geq 2 \lambda-\lambda / 2=3 \lambda / 2$ by (2.10). Also, $\alpha_{n-1}^{\prime}=$ $\left[2 \lambda,-1 / B\left(u_{1}\right), \ldots\right] \geq 3 \lambda / 2$, since $\alpha_{n-1}^{\prime}$ is reduced. Hence,

$$
m_{n-1} \geq 3 \lambda / 2-2 / 3 \lambda>h_{q}+0.3, \quad q \geq 7,
$$

as a calculation shows. It follows that

$$
M\left(\alpha_{0}\right) \geq h_{q}+0.3, \quad q \geq 7,
$$

for $\alpha_{0}$ in this class.

We may now assume all $t_{i}=1$. Define two periodic $\lambda$ CF of period $p=$ $2 s+1$ :

$$
\begin{gathered}
\beta_{0}=\left[2 \lambda,-1 / B(s),-1 / 2 \lambda,-1 / B(s-1), \beta_{0}\right]=\beta_{p}, \\
\gamma_{0}=\left[2 \lambda,-1 / B(s-1),-1 / 2 \lambda,-1 / B(s),-1 / \gamma_{0}\right]=\gamma_{p} .
\end{gathered}
$$

Note that $\gamma_{0}=\beta_{s+1}$, so that $\beta_{0} \sim \gamma_{0}$.

Let

$$
\beta_{0}=\lambda+\delta_{0}, \quad \delta_{0}=\left[\lambda,-\frac{1}{B(s)},-\frac{1}{2 \lambda},-\frac{1}{B(s-1)},-\frac{1}{\beta_{0}}\right],
$$

and let $P_{i} / Q_{i}, i \geq 0$, be the convergents of $\delta_{0} . P_{i}$ and $Q_{i}$ satisfy the recurrence (2.1), and we calculate certain convergents explicitly. Recall $q=2 l-1$. When $2 \leq j \leq s$, the recurrence (2.1) has constant coefficients and we solve for

$$
Q_{j}=A \zeta^{j}+B \zeta^{-j}, \quad \text { where } \zeta=2^{-1}\left(\lambda+\left(\lambda^{2}-4\right)^{1 / 2}\right)=e^{\pi i / q} \text {. }
$$

Hence,

$$
Q_{0}=A+B=1, \quad Q_{1}=A \zeta+B \zeta^{-1}=\lambda
$$

yielding

$$
\begin{gathered}
A=-\zeta /\left(\zeta^{-1}-\zeta\right), \quad B=\zeta^{-1}\left(\zeta^{-1}-\zeta\right), \\
\left(\zeta^{-1}-\zeta\right) Q_{j}=-\zeta^{j+1}+\zeta^{-j-1}=-2 i \sin \pi(j+1) / q .
\end{gathered}
$$

In particular, put $j=s-2=l-4$ :

$$
\left(\zeta^{-1}-\zeta\right) Q_{s-2}=-2 i \sin \pi \frac{l-3}{2 l-1}=-2 i \cos \frac{5 \pi}{2 q} .
$$

Let $\omega=e^{\pi i / 2 q}$; note $\zeta=\omega^{2}, \omega+\omega^{-1}=2 \cos \pi / 2 q, \omega^{2}+\omega^{-2}=\lambda, \omega^{4}+\omega^{-4}=$ $\lambda^{2}-2$. Hence,

$$
\begin{aligned}
2 \cos 5 \pi / q & =\omega^{5}+\omega^{-5}=\left(\omega+\omega^{-1}\right)\left(\omega^{4}-\omega^{2}+1-\omega^{-2}+\omega^{-4}\right) \\
& =\left(\omega+\omega^{-1}\right)\left(\lambda^{2}-\lambda-1\right) .
\end{aligned}
$$

Also, $\zeta^{-1}-\zeta=-2 i \sin \pi / q$. Therefore,

$$
Q_{s-2}=\left(\lambda^{2}-\lambda-1\right) \Omega,
$$


with the abbreviation

$$
\Omega=\frac{\omega+\omega^{-1}}{2 \sin \pi / q}=\frac{1}{2 \sin \pi / 2 q} .
$$

This illustrates the calculation. Similarly we find

$$
Q_{s-1}=(\lambda-1) \Omega,
$$

and by applying the recurrence $(2.1)$ we derive further

$$
\begin{gathered}
Q_{s}=\Omega, \quad Q_{s+1}=(\lambda+1) \Omega, \quad Q_{s+2}=\left(\lambda^{2}+\lambda-1\right) \Omega, \\
Q_{s+3}=\left(\lambda^{3}+\lambda^{2}-2 \lambda-1\right) \Omega .
\end{gathered}
$$

Next we consider $Q_{j}$ for $s+2 \leq j \leq 2 s$. Write $Q_{j}^{\prime}=Q_{s+j+2}$, so now $0 \leq j \leq s-2$. $Q_{j}^{\prime}$ satisfies the same recurrence as $Q_{j}$ with initial values $Q_{0}^{\prime}=Q_{s+2}, Q_{1}^{\prime}=Q_{s+3}$. Solving, we find

$$
\left(\zeta^{-1}-\zeta\right) Q_{j}^{\prime}=-Q_{s+3}\left(\zeta^{j}-\zeta^{-j}\right)+Q_{s+2}\left(\zeta^{j-1}-\zeta^{-j+1}\right)
$$

$0 \leq j \leq s-2$. For example, set $j=s-2=l-4$. Then, $\zeta^{s-2}-\zeta^{-s+2}=$ $2 i \cos 7 \pi / 2 q$ and $\zeta^{s-3}-\zeta^{-s+3}=2 i \cos 9 \pi / 2 q$. The values of the cosines are calculated as in (3.19). Using $\zeta^{-1}-\zeta=-2 i \sin \pi / q$ and the values (3.22), we get

$$
\begin{aligned}
Q_{2 s}= & Q_{s-1}^{\prime} \\
= & \frac{\Omega\left(\omega+\omega^{-1}\right)}{2 \sin \pi / q}\left\{\left(\lambda^{3}+\lambda^{2}-2 \lambda-1\right)\left(\lambda^{3}-\lambda^{2}-2 \lambda+1\right)\right. \\
& \left.\quad-\left(\lambda^{2}+\lambda-1\right)\left(\lambda^{4}-\lambda^{3}-3 \lambda^{2}+2 \lambda+1\right)\right\} \\
= & \frac{\left(\omega+\omega^{-1}\right)^{2}}{4 \sin ^{2} \pi / q} \lambda=\frac{(\lambda+2) \lambda}{4-\lambda^{2}}=\frac{\lambda}{2-\lambda} .
\end{aligned}
$$

In this same way one can derive $Q_{2 s-1}=\left(\lambda^{2}-2\right) /(2-\lambda)$.

To calculate $P_{j}$, we note that

$$
P_{i}=Q_{i+1}, \quad 0 \leq i \leq s-1 .
$$

$P_{s}, \ldots, P_{s+3}$ are calculated by the recurrence $(2.1)$. We now use the analogues of (3.23), (3.24) to get $P_{2 s}, P_{2 s-1}$.

In summary, we now have

$$
\begin{aligned}
& Q_{s-2}=\left(\lambda^{2}-\lambda-1\right) \Omega, \quad Q_{s-1}=(\lambda-1) \Omega, \quad Q_{s}=\Omega \\
& P_{s-2}=Q_{s-1}, \quad P_{s-1}=\Omega, \quad P_{s}=\Omega, \quad \text { where } \Omega=1 /(2 \sin (\pi / 2 q)) \\
& P_{2 s-1}=\left(\lambda^{3}-2 \lambda^{2}+\lambda\right) \omega, \quad P_{2 s}=\left(\lambda^{2}-2 \lambda+2\right) \omega \\
& Q_{2 s-1}=\left(\lambda^{2}-2\right) \omega, \quad Q_{2 s}=\lambda \omega, \quad \text { where } \omega=1 /(2-\lambda) .
\end{aligned}
$$


This gives

$$
\begin{aligned}
& \beta_{0}-\lambda=\delta_{0}=\frac{P_{2 s+1}}{Q_{2 s+1}}=\frac{\beta_{0} P_{2 s}-P_{2 s-1}}{\beta_{0} Q_{2 s}-Q_{2 s-1}}, \\
& \beta_{0}^{2}-(3 \lambda-2) \beta_{0}+2 \lambda^{2}-2 \lambda-1=0, \\
& \beta_{0}=\frac{3 \lambda}{2}-1+\left(1+\left(1-\frac{\lambda}{2}\right)^{2}\right)^{1 / 2} ;
\end{aligned}
$$

we take the plus sign for the square root, since $\beta_{0} \geq 2 / \lambda>1$ from (3.9).

The evaluation of $\gamma_{0}$ is similar:

$$
\gamma_{0}-\lambda=\left[B(s),-\frac{1}{\beta_{0}}\right]=\frac{\beta_{0}-(\lambda-1)}{\beta_{0}(\lambda-1)-\left(\lambda^{2}-\lambda-1\right)} .
$$

At this point, it is convenient to introduce

$$
\rho, \rho^{*}=1-\frac{\lambda}{2} \pm\left(1+\left(1-\frac{\lambda}{2}\right)^{2}\right)^{1 / 2}
$$

so that $\rho \rho^{*}=-1$. Then,

$$
\beta_{0}=\lambda-\rho^{*}
$$

Substituting in (3.27),

$$
\gamma_{0}=\lambda+\frac{\rho^{*}-1}{\rho^{*}(\lambda-1)-1}=\lambda+\rho .
$$

The reverse $\beta_{p-1}^{\prime}$ can be extended to a periodic $\lambda \mathrm{CF}$ of period $p$ with a decrease in value. We denote this fraction by $\beta_{p-1}^{\prime}$ also. Hence

$$
\begin{gathered}
\beta_{t p-1}^{\prime}=\left[\overline{B(s-1)-\frac{1}{2 \lambda},-\frac{1}{B(s)},-\frac{1}{2 \lambda}} ;-\frac{1}{B(s-1)}\right]=\frac{1}{2 \lambda-\gamma_{0}}, \\
\gamma_{t p-1}^{\prime}=\frac{1}{2 \lambda-\beta_{0}} .
\end{gathered}
$$

These values enable us to calculate (see $(2.4)$ )

$$
\begin{aligned}
& m_{t p-1}\left(\beta_{0}\right)=\beta_{t p}-\frac{1}{\beta_{t p-1}^{\prime}}=\beta_{0}-\left(2 \lambda-\gamma_{0}\right) \\
&=\lambda-\rho^{*}+\lambda+\rho-2 \lambda \\
&=2\left(1+\left(1-\frac{\lambda}{2}\right)^{2}\right)^{1 / 2}=h_{q} \\
& m_{t p-1}\left(\gamma_{0}\right)=\gamma_{0}-\left(2 \lambda-\beta_{0}\right)=h_{q} .
\end{aligned}
$$

On the other hand, if $v \not \equiv 0(\bmod p), m_{v-1}\left(\beta_{0}\right)<\beta_{v}=\lambda-\cdots<\lambda<h_{q}$. So,

$$
\varlimsup_{n \rightarrow \infty} m_{n-1}\left(\beta_{0}\right)=h_{q}=\varlimsup_{n \rightarrow \infty} m_{n-1}\left(\gamma_{0}\right) \text {, }
$$


that is,

$$
M\left(\beta_{0}\right)=M\left(\gamma_{0}\right)=h .
$$

Next, we wish to show that $\beta_{0}$ and $\gamma_{0}$ are unique up to $G$-equivalence. Recall that $q \geq 7$, so $s \geq 2$. Define

$$
\begin{array}{cc}
\gamma_{0}^{*}=\left[2 \lambda,-\frac{1}{B(s-1)},-\frac{1}{T}\right], & T=\left[2 \lambda,-\frac{1}{B\left(l_{1}\right)}, \ldots\right], \\
\beta_{0}^{*}=\left[2 \lambda,-\frac{1}{B(s)},-\frac{1}{T}\right], & T=\left[2 \lambda,-\frac{1}{B\left(k_{1}\right)}, \ldots\right] .
\end{array}
$$

We shall show that every $\alpha_{0} \nsim \beta_{0}$ can be replaced by $\beta_{0}^{*}$ or $\gamma_{0}^{*}$ with a decrease in $M\left(\alpha_{0}\right)$.

Consider $\gamma_{0}^{*}$. Since it is reduced, we have $l_{j} \leq s, l_{j}+l_{j+1} \leq 2 s-1, j \geq 1$, by conditions (3.1) and (3.5). Replace $l_{j} \leq s-1$ by $l_{j}=s-1$; this decreases $\gamma_{0}^{*}$. We say the sequence $\left\{l_{j}\right\}$ is alternating if the entries $s-1$ and $s$ occur in succession. Clearly, if $\lambda \mathrm{CF} \gamma_{0}^{*}$ ends in an infinite alternating sequence, then $\gamma_{0}^{*} \sim \gamma_{0}$.

Suppose, on the contrary, that for some odd $t$ the sequence $l_{1}=s, l_{2}, \ldots$, $l_{t+1}=s-1$ is alternating, but $\left(l_{t+2}, l_{t+3}, \ldots, l_{t+k+3}\right)=(s, s-1, \ldots, s-1, s)$. There are $k$ entries $s-1$. If $k$ is odd, we can replace every other $s-1$ by $s$ to obtain an alternating sequence. Suppose $k$ is even, $k=4$, say. Then $\left(l_{t+3}, \ldots, l_{t+6}\right)$ can be replaced by $(s-1, s, s-1, s-1)$. Thus, the sequence we must treat is $(s, s-1, s-1, s)$, and we wish to replace it by $(s, s-1, s, s-1)$. This applies to any even $k$.

What we must prove is that

$$
\left[B(s-1),-\frac{1}{2 \lambda},-\frac{1}{B(s)},-\frac{1}{U}\right]>\left[B(s),-\frac{1}{2 \lambda},-\frac{1}{B(s-1)},-\frac{1}{U}\right],
$$

where $U=\left[2 \lambda,-1 / B\left(l_{1}\right),-1 / V\right]=\lambda+[\lambda, \ldots] \geq \lambda+2 / \lambda>\lambda+1, U<2 \lambda$. By writing the left member as $[\lambda,-1 / B(s-2), \ldots]$, and similarly for the right member, and repeating the process, we eventually bring $(*)$ to the form

$$
\left[\lambda,-\frac{1}{B(s)},-\frac{1}{U}\right]>\left[0,-\frac{1}{2 \lambda},-\frac{1}{B(s-1)},-\frac{1}{U}\right] \text {. }
$$

Since $U>\lambda+1$,

$$
\left[B(s),-\frac{1}{U}\right]>\left[B(s),-\frac{1}{\lambda+1}\right]>\frac{2}{\lambda},
$$

from which it follows that the left member of $(* *)$ is positive. But

$$
[2 \lambda,-1 / B(s-1),-1 / U]>\lambda+[B(s),-1 / U]>0,
$$

so the right member of $(* *)$ is negative. This establishes $(*)$. We have shown that $\gamma_{0}^{*} \geq \gamma_{0}$. Similarly, $\beta_{0}^{*} \geq \beta_{0}$. 
If $n$ is an index for which $\gamma_{n}^{*}=[2 \lambda,-1 / B(s-1), \ldots]$, then by the previous reasoning

$$
\gamma_{n}^{*} \geq \gamma_{0}=\lambda+\rho, \quad \beta_{n}^{*} \geq \beta_{0}=\lambda-\rho^{*} .
$$

Now,

$$
\begin{aligned}
\left(\gamma_{n-1}^{*}\right)^{\prime} & =\left[B\left(l_{k}\right),-\frac{1}{2 \lambda},-\frac{1}{B\left(l_{k-1}\right)},-\frac{1}{W}\right] \\
& \geq\left[B(s),-\frac{1}{2 \lambda},-\frac{1}{B(s-1)},-\frac{1}{W}\right],
\end{aligned}
$$

where $W=\left[2 \lambda,-1 / B\left(l_{k-2}\right), \ldots\right]$, with a finite alternating sequence $l_{k-2}$, $l_{k-3}, \ldots, l_{1}$. This can be extended to an infinite alternating sequence with a decrease in the value of $W$. Hence,

$$
\left(\gamma_{\lambda-1}^{*}\right)^{\prime} \geq \beta_{1}^{*}=\frac{1}{2 \lambda-\beta_{0}^{*}} .
$$

It follows that

$$
\begin{aligned}
m_{n-1}\left(\gamma_{n}^{*}\right) & =\gamma_{n}^{*}-\frac{1}{\left(\gamma_{n-1}^{*}\right)^{\prime}} \geq \gamma_{0}-\left(2 \lambda-\beta_{0}^{*}\right) \\
& \geq \gamma_{0}+\beta_{0}-2 \lambda=h_{q} ;
\end{aligned}
$$

see (3.30). Similarly,

$$
m_{n-1}\left(\beta_{n}^{*}\right) \geq h_{q} .
$$

On the other hand, if $r_{n}=1$, we have $m_{n-1}\left(\beta_{n}\right)<\lambda-\cdots<h_{q}$. We have proved

$$
M\left(\beta_{0}^{*}\right)=\varlimsup_{\nu \rightarrow \infty} m_{\nu}\left(\beta_{0}^{*}\right) \geq M\left(\beta_{0}\right)=h_{q}, \quad M\left(\gamma_{0}^{*}\right) \geq h_{q},
$$

equality occurring if and only if $\beta_{0}^{*}, \gamma_{0}^{*} \sim \beta_{0}$.

Putting (3.13), (3.15), (3.35), and (3.36) together, we get

Lemma 2. If $\alpha_{0}$ has all $\varepsilon_{\nu}=-1$ and $r_{n}=2$, then

$$
m_{n-1}\left(\alpha_{0}\right) \geq h_{q} \text {. }
$$

Hence,

$$
M\left(\alpha_{0}\right) \geq h_{q}, \quad q \geq 5,
$$

with equality if and only if $\alpha_{0} \sim \rho$.

The last statement follows since $r_{n}=2$ must occur infinitely often.

To complete the proof of Theorem 1, we proceed as follows. If $\varepsilon_{\mu}=1$ occurs in $\alpha_{0}$ only a finite number of times, we may assume it never occurs; then by (3.2), $r_{\mu} \geq 2$ infinitely often. Hence $M\left(\alpha_{0}\right) \geq h_{q}$ by Lemma 2 , with the cases of equality mentioned there. So we now assume $\varepsilon_{\mu}=1$ occurs infinitely 
often but not always. We look for the largest block of terms with $\varepsilon=-1$, i.e., bounded by $\varepsilon=+1$ at both ends. Denote this block by

$$
\alpha_{\mu \nu}=\left[r_{\mu} \lambda,-\frac{1}{r_{\mu+1} \lambda}, \ldots, \frac{1}{r_{\nu} \lambda}\right], \quad \varepsilon_{\mu}=\varepsilon_{\nu+1}=1 .
$$

The terms with $r_{t}=1$ yield only $m_{t-1}<\lambda<h_{q}, \mu+1 \leq t \leq \nu$. So let $r_{n}=2$ for an $n$ with $\mu+1 \leq n \leq \nu$. If $\alpha_{\mu \nu}$ does not end in $B(s)$, $-1 / 2 \lambda,-1 / B(s)$, we can adjoin $U$ with all $\varepsilon=-1$ so that $\left[\alpha_{\mu \nu},-1 / U\right]$ is reduced: for example, we could take a periodic $U=[2 \lambda,-1 / 2 \lambda, \ldots]$. Then by (2.2), $\alpha_{n}>\alpha_{n \nu}>\left[\alpha_{n \nu},-1 / U\right]$. Similarly, $\alpha_{n-1}^{\prime}>\alpha_{n-1, \mu}^{\prime}>\left[\alpha_{n-1, \mu}^{\prime},-1 / V\right]$, where $V=\left[r_{\mu-1}^{\prime} \lambda,-1 / r_{\mu-2}^{\prime} \lambda, \ldots,-1 / r_{1}^{\prime} \lambda\right]$ is chosen so that $\left[\alpha_{n-1, \mu}^{\prime},-1 / V\right]$ is reduced. Then, $\delta_{0}:=\left[V^{\prime},-1 / \alpha_{\mu \nu},-1 / U\right]$ has all $\varepsilon=-1$ and is reduced. By Lemma 2,

$$
m_{n-1}\left(\alpha_{0}\right) \geq m_{n-1}\left(\delta_{0}\right) \geq h_{q}, \quad r_{n} \geq 2
$$

It follows that

$$
M\left(\alpha_{0}\right) \geq M\left(\delta_{0}\right) \geq h_{q}
$$

When $\alpha_{\mu \nu}$ ends with $B(s),-1 / 2 \lambda,-1 / B(s)$, there is no $U$ satisfying the required conditions because of (3.3). We derive successively, using the values (3.25):

$$
\begin{gathered}
\alpha_{0}=[\ldots,-1 / B(s), 1 / T], \\
{\left[B(s), \frac{1}{T}\right]=\frac{T P_{s-1}+P_{s-2}}{T Q_{s-1}+Q_{s-2}}>\frac{1}{\lambda-1},} \\
{\left[2 \lambda,-\frac{1}{B(s)}, \frac{1}{T}\right]>\lambda+1,} \\
{\left[B(s),-\frac{1}{2 \lambda},-\frac{1}{B(s)}, \frac{1}{T}\right]>\left[B(s),-\frac{1}{\lambda+1}\right]=\frac{2}{\lambda},} \\
{\left[2 \lambda,-\frac{1}{B(s)}, \ldots, \frac{1}{T}\right]>\left[2 \lambda,-\frac{\lambda}{2}\right]=\frac{3 \lambda}{2},}
\end{gathered}
$$

and finally

$$
\alpha_{n} \geq \eta_{n}:=\left[2 \lambda,-\frac{1}{B\left(l_{1}\right)}, \ldots,-\frac{1}{B\left(l_{k}\right)},-\frac{1}{3 \lambda / 2}\right] .
$$

We assign $l_{j}=s$ or $s-1$ in alternation, so that $\eta_{n}$ is of the form $\beta_{0}^{*}$ in (3.34) or $\gamma_{0}^{*}$ in (3.33); then from (3.37), (3.36) we again get (3.39), (3.40).

The final case is: all $\varepsilon=+1$. If $1 / r_{n} \lambda$ occurs with $r_{n} \geq 2$, then $m_{n-1} \geq$ $\alpha_{n}>2 \lambda>h_{q}$. When $1 / r_{n} \lambda$ occurs infinitely often, we get

$$
M(\alpha)=\varlimsup_{n \rightarrow \infty} m_{n-1}(\alpha) \geq 2 \lambda>h_{q} .
$$

Otherwise, we may assume $1 / r \lambda, r \geq 2$, never occurs and

$$
\alpha_{n}=\left[\lambda, \frac{1}{\lambda}, \ldots\right]=\left[\lambda, \frac{1}{\alpha_{n}}\right]=\frac{1}{2}\left(\lambda+\left(\lambda^{2}+4\right)^{1 / 2}\right)=: \mu .
$$


So,

$$
m_{n-1}=\mu+\frac{1}{\mu}=\left(\lambda^{2}+4\right)^{1 / 2}>h_{q},
$$

as a small calculation shows, and this implies

$$
M\left(\alpha_{0}\right)>h_{q} .
$$

In all cases, then, $M\left(\alpha_{0}\right)$ is bounded below by $h_{q}$, with the cases of equality stated in (3.12), (3.37), (3.38). This completes the proof of Theorem 1.

\section{The local Hurwitz constant}

In this section we shall consider the local Hurwitz constant, i.e., $m_{i}\left(\alpha_{0}\right)$. Our object is to compare $m_{i}$ with $h_{q}$.

We first use a geometric method. Let $\alpha$ be $G$-irrational. The Ford circle $C_{n}$ is defined by

$$
C_{n}:\left|z-\left(\frac{P_{n}}{Q_{n}}+\frac{i}{2 Q_{n}^{2}}\right)\right|=\frac{1}{2 Q_{n}^{2}},
$$

where $P_{n} / Q_{n}$ are the convergents of $\alpha$. Different $C_{n}$ do not overlap; $C_{n}$ and $C_{m}$ are tangent externally if and only if $m=n+1$ or $n-1$. These assertions follow easily from the determinant condition (2.2). Also from (2.3), (2.12), with $\alpha=\left[r_{0} \lambda, \varepsilon_{1} / r_{1} \lambda, \ldots\right]$, we have

$$
\operatorname{sgn}\left(\alpha-\frac{P_{n-1}}{Q_{n-1}}\right)= \pm \operatorname{sgn}\left(\alpha-\frac{P_{n}}{Q_{n}}\right)
$$

according as $\varepsilon_{n+1}=-1$ or +1 .

Suppose $\varepsilon_{n+1}=-1$. Then $\alpha$ is on the same side of both $P_{n} / Q_{n}$ and $P_{n-1} / Q_{n-1}$. It follows that

$$
\left|\alpha-\frac{P_{i}}{Q_{i}}\right|>\frac{1}{Q_{i}^{2}} \text { for } i=n-1 \text { or } n \text {. }
$$

Equality is impossible because $P_{i} / Q_{i}$ is $G$-rational, but $\alpha$ is $G$-irrational.

Next suppose $\varepsilon_{n+1}=1$. Then $\alpha$ lies between $P_{n} / Q_{n}$ and $P_{n-1} / Q_{n-1}$. Let $(i, j)$ be a permutation of $(n-1, n)$. Then,

$$
\left|\alpha-\frac{P_{i}}{Q_{i}}\right|<\frac{1}{2 Q_{i}^{2}}, \quad\left|\alpha-\frac{P_{j}}{Q_{j}}\right|>\frac{1}{2 Q_{j}^{2}} .
$$

Equality can occur only if $\alpha$ coincides with the real projection of the point of tangency of the Ford circles, which is impossible because $\alpha$ is $G$-irrational. Hence,

Theorem 2. If $\varepsilon_{n+1}=1$, we have $m_{n-1}>2, m_{n}<2$, or $m_{n-1}<2, m_{n}>2$.

An elegant algebraic proof of this theorem in the rational case $(q=3)$ was given by $\mathrm{K}$. Th. Vahlen [6].

Theorem 2 holds for all $q \geq 4$, even or odd. Since $h_{q}=2$ when $q$ is even, it provides an estimate of the desired type for even $q$. We now concentrate on odd $q$. 
Theorem 3. Let $q$ be odd. If $r_{n} \geq 2$ and $\varepsilon_{n-1}=1$, then $m_{n-1} \geq h_{q}$.

Theorem 3 is a special case of (3.39).

If we drop the assumption $r_{n} \geq 2$, we can have two consecutive $m_{i}<h_{q}$, as we see from the following example: let

$$
\begin{gathered}
\lambda=\lambda_{7}=1.80, \ldots, r_{n-1}=4, \\
\alpha_{0}=[\ldots,-1 / 4 \lambda, 1 / \lambda,-1 / \lambda,-1 / \lambda, \ldots],
\end{gathered}
$$

for which $m_{n}<\lambda, m_{n-1}<1.97<2$. We make further assumptions on the $\varepsilon_{i}$.

Theorem 4. Let $q$ be odd. If $\varepsilon_{n+1}=\varepsilon_{n+2}=1$, then $m_{i} \geq\left(\lambda^{2}+4\right)^{1 / 2}>h_{q}$ for at least one of $i=n-1, n, n+1$.

The proof is modelled after one by M. Fujiwara [2]; see also F. Bagemihl and J. R. McLaughlin [1]. In contradiction to the conclusion

$$
m_{i}(\alpha) \geq\left(\lambda^{2}+4\right)^{1 / 2},
$$

we can assert that

$$
\left|\alpha-\frac{P_{j}}{Q_{j}}\right|>\frac{1}{\left(\lambda^{2}+4\right)^{1 / 2} Q_{j}^{2}}, \quad n-1 \leq j \leq n+1 .
$$

We observe from (2.3) that $\alpha-P_{n-1} / Q_{n-1}$ and $\alpha-P_{n} / Q_{n}$ have opposite signs, in view of $\varepsilon_{n+1}=1$. Hence,

$$
\frac{1}{\left(\lambda^{2}+4\right)^{1 / 2}}\left(\frac{1}{Q_{n-1}^{2}}+\frac{1}{Q_{n}^{2}}\right)<\left|\alpha-\frac{P_{n-1}}{Q_{n-1}}\right|+\left|\alpha-\frac{P_{n}}{Q_{n}}\right|=\frac{1}{Q_{n} Q_{n-1}} .
$$

Write

$$
\left(\lambda^{2}+4\right)^{1 / 2}=u+\frac{1}{u}, \quad u>\lambda
$$

then

$$
\frac{Q_{n}^{2}}{Q_{n-1}^{2}}-\left(\lambda^{2}+4\right)^{1 / 2} \frac{Q_{n}}{Q_{n-1}}+1=\left(\frac{Q_{n}}{Q_{n-1}}-\frac{1}{u}\right)\left(\frac{Q_{n}}{Q_{n-1}}-u\right)<0 .
$$

Now $Q_{n} / Q_{n-1}-1 / u>1-1=0$, so $Q_{n} / Q_{n-1}-u<0$, that is,

$$
\frac{Q_{n}}{Q_{n-1}}<u \text {. }
$$

Hence,

$$
\frac{Q_{n-1}}{Q_{n}}>\frac{1}{u} .
$$

Replacing $n$ by $n+1$ in (4.6)-recall $\varepsilon_{n+2}=1$-we get

$$
\frac{Q_{n+1}}{Q_{n}}<u \text {. }
$$


Therefore,

yielding

$$
u>\frac{Q_{n+1}}{Q_{n}}=r_{n+1} \lambda+\varepsilon_{n+1} \frac{Q_{n-1}}{Q_{n}} \geq \lambda+\frac{Q_{n-1}}{Q_{n}},
$$

$$
\frac{Q_{n-1}}{Q_{n}}<u-\lambda .
$$

But

$$
\left(u-\frac{1}{u}\right)^{2}=\left(u+\frac{1}{u}\right)^{2}-4=\lambda^{2},
$$

and so

$$
\frac{Q_{n-1}}{Q_{n}}<\frac{1}{u},
$$

contradicting (4.7). This completes the proof of Theorem 4.

Note added in proof. In a recent letter Thomas A. Schmidt has pointed out an error in [4] that carries over to the present paper. It can be corrected as follows. Replace the two paragraphs following (1.5) by the following:

We now consider the approximation of a $G$-irrational $\alpha_{0}$ by the convergents $P_{n} / Q_{n}$ of its $\lambda \mathrm{CF}$ (1.4). Note that in (1.1) the fraction $k / m \in G(\infty)$ determines $k$ and $m$ uniquely up to sign, since

$$
\left(\begin{array}{cc}
k & \cdot \\
m & \cdot
\end{array}\right)^{-1}\left(\begin{array}{cc}
k_{1} & \cdot \\
m_{1} & \cdot
\end{array}\right)=\left(\begin{array}{ll}
\cdot & \cdot \\
0 & \cdot
\end{array}\right)= \pm\left(\begin{array}{ll}
1 & \cdot \\
0 & 1
\end{array}\right)
$$

when $k / m=k_{1} / m_{1}$. Thus we write

$$
\alpha_{0}-\frac{P_{n-1}}{Q_{n-1}}=\frac{(-1)^{n-1} \varepsilon_{1} \varepsilon_{2} \cdots \varepsilon_{n}}{m_{n-1} Q_{n-1}^{2}}, \quad m_{n-1}=m_{n-1}\left(\alpha_{0}\right),
$$

and study $m_{n-1}\left(\alpha_{0}\right)$. Clearly,

$$
M\left(\alpha_{0}\right)=\varlimsup_{n \rightarrow \infty} m_{n-1}\left(\alpha_{0}\right), \quad h_{q}^{\prime}=\inf _{\alpha_{0}} M\left(\alpha_{0}\right) .
$$

We call $m_{n}\left(\alpha_{0}\right)$ a local Hurwitz constant.

Similar changes are required in [4]. In particular, Theorem 3 should be eliminated.

\section{BIBLIOGRAPHY}

1. F. Bagemihl and J. R. McLaughlin, Generalization of some classical theorems concerning triples of consecutive convergents to simple continued fractions, J. Reine Angew. Math. 221 (1966), 146-149.

2. M. Fujiwara, Bemerkung zur Theorie der Approximation der irrationalen Zahlen durch rationale Zahlen, Tôhoku Math. J. 14 (1918), 109-115.

3. A. Haas and C. Series, The Hurwitz constant and Diophantine approximation on Hecke groups, J. London Math. Soc. 34 (1986), 219-234.

4. J. Lehner, Diophantine approximation on Hecke groups, Glasgow Math. J. 27 (1985), 117127. 
5. D. Rosen, A class of continued fractions associated with certain properly discontinuous groups, Duke Math. J. 21 (1954), 549-564.

6. K. Th. Vahlen, Ueber Näherungswerthe und Kettenbrüche, J. Reine Angew. Math. 115 (1895), 221-233.

314 N. Sharon Way, Jamesburg, New Jersey 08831 